\begin{tabular}{|c|c|}
\hline Title & Construction of a domain that fails the global boundary Harnack principle via the Helmholtz equation \\
\hline Author(s) & A ikawa, Hiroaki \\
\hline Citation & $\begin{array}{l}\text { Journal of the London Mathematical Society. Second series, 93(3), 721-740 } \\
\text { https://doi.org/10.1112 } / \mathrm{Ims} \not \mathrm{dw} 016\end{array}$ \\
\hline Issue Date & $2016-06$ \\
\hline Doc URL & http:/hdl.handle.net/2115/66353 \\
\hline Rights & $\begin{array}{l}\text { This is the accepted version of the following article: A ikawa, H. (2016), Construction of a domain that fails the global } \\
\text { boundary Harnack principle via the Helmholtz equation. Journal of the London Mathematical Society, 93: } 721-740 \text {, } \\
\text { which has been published in final form at doi:10.1112 } \not 1 \mathrm{~ms} / \mathrm{d} \text { dw016. }\end{array}$ \\
\hline Type & article (author version) \\
\hline File Information & prhe.pdf \\
\hline
\end{tabular}

Instructions for use 


\title{
CONSTRUCTION OF A DOMAIN THAT FAILS THE GLOBAL BOUNDARY HARNACK PRINCIPLE VIA THE HELMHOLTZ EQUATION
}

\author{
HIROAKI AIKAWA
}

\begin{abstract}
Aвstract. We show the sharpness of the modulus of continuity of a function $f$ for which the domain lying above the graph of $f$ satisfies the global boundary Harnack principle, with the aid of precise estimates of the Poisson integrals with respect to the Helmholtz equation in the half space.
\end{abstract}

\section{INTRODUCTION}

Ever since the pioneering works of Dahlberg [Dah77], Ancona [Anc78] and Wu [Wu78] for Lipschitz domains, a large amount of work has been devoted to the study of the boundary Harnack principle for nonsmooth domains such as Lipschitz domains, nontangentially accessible domains, uniform domains, John domains, Hölder domains, and domains satisfying the quasihyperbolic boundary condition; see Jerison-Kenig [JK82] for nontangentially accessible domains, Bañuelos-Bass-Burdzy [BBB91], [BB91], [BB94] for Hölder domains, and the author [Aik01] and [Aik04] for uniform domains and inner uniform domains. The validity of the boundary Harnack principle heavily depends on the geometry of the domain. This is a sharp contrast with the fact that the boundary Harnack principle with respect to a non-local operator such as the fractional Laplacian holds for arbitrary domains (see Bogdan [Bog97] and Song-Wu [SW99]). In this paper, we show the sharpness of the modulus of continuity of a function $f$ for which the domain lying above the graph of $f$ satisfies the global boundary Harnack principle with respect to classical harmonic functions.

First we recall that there are two different types of the boundary Harnack principle. Let $D$ be a domain in $\mathbb{R}^{n}$ with $n \geq 2$. Consider a pair $(V, K)$ of a bounded open set $V \subset \mathbb{R}^{n}$ and a compact set $K \subset \mathbb{R}^{n}$ such that

$$
K \subset V, K \cap D \neq \emptyset \text { and } K \cap \partial D \neq \emptyset .
$$

Definition 1.1. We say that $D$ enjoys the global boundary Harnack principle if for each pair $(V, K)$ with (1.1) the following property holds: If $u$ and $v$ are positive superharmonic functions on $D$ such that

(i) $u$ and $v$ are bounded, positive and harmonic in $V \cap D$,

(ii) $u$ and $v$ vanish on $V \cap \partial D$ outside a polar set, then

$$
\frac{u(x) / u(y)}{v(x) / v(y)} \leq C \quad \text { for } x, y \in K \cap D
$$

where $C$ depends only on $D, V$ and $K$.

There is another type of boundary Harnack principle, i.e., the scale invariant boundary Harnack principle. By $B(x, r)$ we denote the open ball with center at $x$ and radius $r$.

2010 Mathematics Subject Classification. 31B25,35J67.

Key words and phrases. Boundary Harnack principle, modulus of continuity, Helmholtz equation.

This work was supported in part by JSPS KAKENHI Grant Numbers 25287015 and 25610017. 
Definition 1.2. We say that $D$ enjoys the scale invariant boundary Harnack principle if there exist constant $C>1$ and $r_{0}>0$ with the following property: If $\xi \in \partial D, 0<r \leq r_{0}$ and

(i) $u$ and $v$ are bounded, positive harmonic functions in $B(\xi, C r) \cap D$,

(ii) $u$ and $v$ vanish on $B(\xi, C r) \cap \partial D$ except for a polar set, then

$$
\frac{u(x) / u(y)}{v(x) / v(y)} \leq C \quad \text { for } x, y \in B(\xi, r) \cap D .
$$

The scale invariant boundary Harnack principle is a property much stronger than the global boundary Harnack principle. It is classical that the scale invariant boundary Harnack principle holds for a Lipschitz domain ([Anc78] and [Wu78]) and for an NTA-domain ([JK82]). In [Aik01] the author showed the scale invariant boundary Harnack principle for a uniform domain; and in [Aik04] the converse, i.e., the uniformity of $D$ is characterized by the validity of the scale invariant boundary Harnack principle on $D$, under a suitable additional assumption on $D$. We note that the quantitative nature of the scale invariant boundary Harnack principle played an important role.

The global boundary Harnack principle is a rather weak qualitative property, which holds for very nasty domains such as a John domain and a domain satisfying the quasihyperbolic boundary condition (Bass-Burdzy [BB91]; see also [Aik14] and [Aik15]). More precise results are known for a domain whose boundary is locally given by the graph of a continuous function in $\mathbb{R}^{n-1}$. Let $\psi(t)$ be a nondecreasing continuous function for $t \geq 0$ with $\psi(0)=0$. We say that a function $f$ in $\mathbb{R}^{n-1}$ is $\psi$-Hölder continuous if

$$
\left|f\left(x^{\prime}\right)-f\left(y^{\prime}\right)\right| \leq C \psi\left(\left|x^{\prime}-y^{\prime}\right|\right) \quad \text { for } x^{\prime}, y^{\prime} \in \mathbb{R}^{n-1}
$$

where $C>0$ is independent of $x^{\prime}, y^{\prime} \in \mathbb{R}^{n-1}$. We say that a bounded domain in $\mathbb{R}^{n}$ is a $\psi$-Hölder domain if its boundary is locally given by the graph of a $\psi$-Hölder continuous function in $\mathbb{R}^{n-1}$. If $0<\alpha \leq 1$, then a $t^{\alpha}$-Hölder domain is simply called an $\alpha$-Hölder domain. A 1-Hölder domain is called a Lipschitz domain. In case $n=2$, conformal mappings are available, and hence the global boundary Harnack principle holds for every $\psi$-Hölder domain no matter how bad $\psi$ is. So we let $n \geq 3$ for the moment.

Bass-Burdzy [BB91] proved probabilistically the global boundary Harnack principle for an $\alpha$-Hölder domain in case $1 / 2<\alpha \leq 1$, and then Bañuelos-Bass-Burdzy [BBB91] extended the range of $\alpha$ to $0<\alpha \leq 1$. In the opposite direction, Bass-Burdzy ([BB91, Proposition 5.3]) constructed a domain lying above the graph of a continuous function (but not a Hölder continuous) for which the global boundary Harnack principle fails. Unfortunately, their construction was involved and no good control of modulus of continuity was obtained. So, sharp criterion of modulus of continuity of the boundary function for the validity of the global boundary Harnack principle had remained open.

In the previous paper [Aik14] we proved an extended Harnack inequality with exceptional sets, which asserts that a Harnack inequality holds even if a small (but non-polar) exceptional set lies in the Harnack chain. This is a generalization of [BB92, Lemma 2.14], which played a crucial role in [BBB91] to extend the range of the Hölder exponent $\alpha$. With the aid of this new Harnack inequality, we showed that the global boundary Harnack principle holds for a $\psi$-Hölder domain with modulus of continuity $\psi$ weaker than Hölder continuity. For $\alpha>0$ let $\psi_{\alpha}(t)=(-\log t)^{-\alpha}$ for $0<t<1 / e^{\alpha+1}$ and extend it by constant for $t \geq 1 / e^{\alpha+1}$. Sometimes $\psi_{\alpha}$ is referred to as $\log$-Hölder continuity of order $\alpha$. 
Theorem A ([Aik14, Theorem 1.3]). Let $n \geq 3$. If a nondecreasing continuous function $\psi$ satisfies the Dini condition:

$$
\int_{0} \frac{\psi(t)}{t} d t<\infty
$$

with an additional assumption $\lim \sup _{t \rightarrow 0} \psi(M t) / \psi(t)<M$ for some $M>1$, then every $\psi$ Hölder domain satisfies the global boundary Harnack principle. In particular, if $\alpha>1$, then every $\psi_{\alpha}$-Hölder domain satisfies the global boundary Harnack principle.

The main purpose of this paper is to show the sharpness of Theorem A.

Theorem 1.1. Let $n \geq 3$. If $0<\alpha<1$, then there exists a $\psi_{\alpha}$-Hölder domain which fails the global boundary Harnack principle.

Theorems A and 1.1 show that that the threshold for the global boundary Harnack principle is $\log$-Hölder continuity of order 1, i.e., it lies in between $\psi_{\alpha}(t)=(-\log t)^{-\alpha}$ of $\alpha>1$ and that of $\alpha<1$. We do not know what happens for $\psi_{1}(t)=(-\log t)^{-1}$.

Let us remark some potential theoretical properties related to the boundary Harnack principle. We can easily generalize the Laplace operator to uniformly elliptic operators of divergence form (see [CFMS81]). However, there is a significant difference between uniformly elliptic operators of divergence form and those of non-divergence form. The threshold for the global boundary Harnack principle with respect to a uniformly elliptic operator of non-divergence form is $1 / 2$ Hölder continuity. In fact, Bass-Burdzy [BB94] showed the following:

(i) If $1 / 2<\alpha \leq 1$, then every $\alpha$-Hölder domain satisfies the global boundary Harnack principle with respect to a uniformly elliptic operator of non-divergence form.

(ii) If $0<\alpha<1 / 2$, then there exist an $\alpha$-Hölder domain and a uniformly elliptic operator $L$ of non-divergence form for which the global boundary Harnack principle with respect to $L$ fails.

The scale invariant boundary Harnack principle immediately implies that the Martin boundary of the domain is homeomorphic to the Euclidean boundary. This is the case for a Lipschitz domain. Actually, Bass-Burdzy [BB93] showed that modulus of continuity slightly worse than Lipschitz is sufficient for the Martin boundary of a domain to be homeomorphic to the Euclidean boundary, and that the critical modulus of continuity is $\psi(t)=t \log \log (1 / t)$. The following table summarizes potential theoretical properties of a domain locally given by the graph of a continuous function $f$ and the critical moduli of continuity of $f$. Here the BHP stands for the boundary Harnack principle. This paper, together with [Aik14], completes the last assertion of the table.

TABLE 1. Potential theoretical properties and critical moduli of continuity

\begin{tabular}{l|l} 
Potential theoretical property & Critical modulus of continuity \\
\hline \hline Scale-invariant BHP & $\psi(t)=t$ \\
Martin boundary = Euclidean boundary & $\psi(t)=t \log \log (1 / t)$ \\
Global BHP for non-divergence operator & $\psi(t)=t^{1 / 2}$ \\
Global BHP for divergence operator & $\psi(t)=(\log (1 / t))^{-1}$ \\
\hline
\end{tabular}

Let us state our methodology for Theorem 1.1. Bass-Burdzy ([BB91, Proposition 5.3]) constructed their domain based on hitting probabilities, or harmonic measures in analytic terminology. We follow basically the same approach as in [BB91], but with the aid of precise estimates of harmonic measure with respect to the Helmholtz equation, which may be of independent 
interest. Roughly speaking we construct a domain by digging the bottom of a cube and make countably many sharp ravines widening out rapidly. See Figure 5 in Section 4 . Heuristically, if the Brownian motion starts at a point near the bottom of a ravine, then the hitting probability of the top is much smaller than that of the side, provided sharpness and widening satisfy a subtle relation. However, the fact that the top of a ravine is always visible from the bottom (although it is very narrow) gives rise to difficulty in the upper estimate of the hitting probability of the top. The precise estimates of harmonic measures with respect to the Helmholtz equation enables us to overcome this difficulty. We do not know whether probabilistic arguments yield such precise estimates.

Now let $n \geq 2$ and $\lambda>0$. We study the Poisson representation for the Helmholtz equation $\left(-\Delta+\lambda^{2}\right) u=0$ in the upper half space $\mathbb{R}_{+}^{n}=\left\{x=\left(x_{1}, \ldots, x_{n}\right): x_{n}>0\right\}$. It is well known that the Green function $G_{\lambda}(x)$ for $-\Delta+\lambda^{2}$ in $\mathbb{R}^{n}$ is given by

$$
G_{\lambda}(x)=\int_{0}^{\infty}(4 \pi t)^{-n / 2} \exp \left(-\lambda^{2} t-\frac{|x|^{2}}{4 t}\right) d t .
$$

Let $P_{\lambda}(x)=-2 \partial G_{\lambda}(x) / \partial x_{n}$. Define the Poisson kernel with respect to $-\Delta+\lambda^{2}$ for $\mathbb{R}_{+}^{n}$ by

$$
P_{\lambda}\left(x ; y^{\prime}\right)=P_{\lambda}\left(x-\left(y^{\prime}, 0\right)\right) \quad \text { for } x \in \mathbb{R}_{+}^{n} \text { and }\left(y^{\prime}, 0\right) \in \partial \mathbb{R}_{+}^{n} .
$$

For simplicity we identify $\partial \mathbb{R}_{+}^{n}$ and $\mathbb{R}^{n-1}$. We have the following Poisson representation for the Helmholtz equation $\left(-\Delta+\lambda^{2}\right) u=0$ in $\mathbb{R}_{+}^{n}$.

Theorem 1.2. Let $f$ be a bounded continuous function on $\partial \mathbb{R}_{+}^{n}$. Then

$$
\begin{aligned}
\left(-\Delta+\lambda^{2}\right) u & =0 \text { in } \mathbb{R}_{+}^{n}, \\
u & =f \text { on } \partial \mathbb{R}_{+}^{n},
\end{aligned}
$$

has a unique bounded solution which is represented as

$$
P_{\lambda}[f](x):=\int_{\mathbb{R}^{n-1}} P_{\lambda}\left(x ; y^{\prime}\right) f\left(y^{\prime}\right) d y^{\prime} .
$$

If $f \equiv 1$ on $\mathbb{R}^{n-1}$, then

$$
P_{\lambda}[1](x)=\exp \left(-\lambda x_{n}\right)
$$

This may be regarded as the harmonic measure with respect to $-\Delta+\lambda^{2}$ of the whole boundary $\partial \mathbb{R}_{+}^{n}$. One may expect that a subset of the boundary has the harmonic measure decaying faster than $\exp \left(-\lambda x_{n}\right)$. The following theorem gives a precise decay estimate.

Theorem 1.3. Let $a>0$. If $x=\left(x^{\prime}, x_{n}\right) \in \mathbb{R}_{+}^{n}$ and $\lambda x_{n} \geq 1$, then

$$
\int_{\left|y^{\prime}-x^{\prime}\right| \geq a} P_{\lambda}\left(x ; y^{\prime}\right) d y^{\prime} \leq \exp \left(-\lambda x_{n}\right) \cdot\left\{C \exp \left(-\frac{\lambda x_{n}}{4}\right)+C\left(\lambda x_{n}\right)^{(n-1) / 2} \exp \left(-\frac{\lambda a^{2}}{3 x_{n}}\right)\right\},
$$

where $C>0$ depends only on the dimension $n$.

Observe that there is a close relationship between harmonic functions in $\mathbb{R}^{n}$ and solutions to the Helmholtz equation in $\mathbb{R}^{n-1}$. More precisely, if $\left(-\Delta^{\prime}+\lambda^{2}\right) u\left(x^{\prime}\right)=0$ in $D^{\prime} \subset \mathbb{R}^{n-1}$ with $\Delta^{\prime}$ being the Laplacian in $\mathbb{R}^{n-1}$, then $u\left(x^{\prime}\right) \cos \left(\lambda x_{n}\right)$ is a harmonic function in $D^{\prime} \times(-\varepsilon, \varepsilon)$ vanishing on $D^{\prime} \times\{-\varepsilon, \varepsilon\}$ with $\varepsilon=\pi /(2 \lambda)$. Hence Theorems 1.2 and 1.3 can be applied to the study of harmonic functions in $D^{\prime} \times(-\varepsilon, \varepsilon)$.

We use the following notation. By the symbol $C$ we denote an absolute positive constant whose value is unimportant and may change from one occurrence to the next. By $C(p, q, \ldots)$ we mean that $C$ depends on $p, q, \ldots$ We often suppress the dependency on the dimension $n$. 
If necessary, we use $C_{0}, C_{1}, \ldots$, to specify constants. We say that $f$ and $g$ are comparable and write $f \approx g$ if two positive quantities $f$ and $g$ satisfies $C^{-1} \leq f / g \leq C$ with some constant $C \geq 1$. The constant $C$ is referred to as the constant of comparison. We have to pay attention for the dependency of the constant of comparison.

Acknowledgments. The author thanks Krzysztof Burdzy for valuable comments.

\section{Proof of Theorems 1.2 AND 1.3}

We frequently use the following maximum principle (or Phragmén-Lindelöf principle) for the Helmholtz equation.

Lemma 2.1. Let $D$ be an arbitrary domain (bounded or unbounded) in $\mathbb{R}^{n}$. If $u$ is a bounded solution to the Helmholtz equation $\left(-\Delta+\lambda^{2}\right) u=0$ in $D$ with vanishing boundary values, then $u \equiv 0$ in $D$.

Proof. If $u$ is a bounded solution to the Helmholtz equation $\left(-\Delta+\lambda^{2}\right) u=0$ in $D$ with vanishing boundary values, then $h\left(x, x_{n+1}\right)=u(x) \cos \left(\lambda x_{n+1}\right)$ is a bounded harmonic function in $D \times(-\pi /(2 \lambda), \pi /(2 \lambda))$ with vanishing boundary values. Hence the Phragmén-Lindelöf principle for harmonic functions gives the lemma.

Let us rewrite the Poisson kernel for the Helmholtz equation by using the modified Bessel kernel of the third kind

$$
K_{v}(z)=\frac{1}{2}\left(\frac{z}{2}\right)^{v} \int_{0}^{\infty} \exp \left(-t-\frac{z^{2}}{4 t}\right) \frac{d t}{t^{\nu+1}},
$$

which has another integral representation

$$
K_{v}(z)=\left(\frac{\pi}{2 z}\right)^{1 / 2} \frac{e^{-z}}{\Gamma(v+1 / 2)} \int_{0}^{\infty} e^{-t} t^{\nu-1 / 2}\left(1+\frac{t}{2 z}\right)^{v-1 / 2} d t
$$

if $v>-1 / 2$. In fact, (2.1) has an analytic continuation to a suitable domain in the complex plane. In this note, however, (2.1) for $z>0$ is sufficient. See [Wat95, 6.22 (15) and 7.30]. For the reader's convenience, a self-contained proof is provided in the appendix.

By a change of variable in (1.2) we have

$$
G_{\lambda}(x)=\frac{1}{2 \pi}\left(\frac{2 \pi|x|}{\lambda}\right)^{1-n / 2} K_{n / 2-1}(\lambda|x|) .
$$

The differentiation under the integral sign in (1.2) yields

$$
P_{\lambda}(x)=\frac{x_{n}}{(4 \pi)^{n / 2}} \int_{0}^{\infty} \exp \left(-\lambda^{2} t-\frac{|x|^{2}}{4 t}\right) \frac{d t}{t^{n / 2+1}}=2 x_{n}\left(\frac{\lambda}{2 \pi|x|}\right)^{n / 2} K_{n / 2}(\lambda|x|) .
$$

We estimate $P_{\lambda}(x)$ by making use of (2.1).

Lemma 2.2. Let $x \in \mathbb{R}_{+}^{n}$ and $y=\left(y^{\prime}, 0\right) \in \partial \mathbb{R}_{+}^{n}$ with $y^{\prime} \in \mathbb{R}^{n-1}$. Then

$$
P_{\lambda}\left(x ; y^{\prime}\right) \leq \frac{x_{n}}{\Gamma\left(\frac{n+1}{2}\right)}\left(\frac{\lambda}{2 \pi}\right)^{(n-1) / 2} \frac{\exp (-\lambda|x-y|)}{|x-y|^{(n+1) / 2}}\left\{\left(\frac{3}{2}\right)^{(n-1) / 2} \Gamma\left(\frac{n+1}{2}\right)+(n-1) !\left(\frac{3}{2 \lambda|x-y|}\right)^{(n-1) / 2}\right\} .
$$

Proof. In view of (2.1) and (2.2) we have

$$
P_{\lambda}\left(x ; y^{\prime}\right)=\frac{x_{n}}{\Gamma\left(\frac{n+1}{2}\right)}\left(\frac{\lambda}{2 \pi}\right)^{(n-1) / 2} \frac{\exp (-\lambda|x-y|)}{|x-y|^{(n+1) / 2}} \int_{0}^{\infty} t^{(n-1) / 2}\left(1+\frac{t}{2 \lambda|x-y|}\right)^{(n-1) / 2} e^{-t} d t .
$$


Letting $\alpha=\lambda|x-y|$, we decompose the last integral into

$$
\int_{0}^{\alpha}+\int_{\alpha}^{\infty} t^{(n-1) / 2}\left(1+\frac{t}{2 \alpha}\right)^{(n-1) / 2} e^{-t} d t
$$

The first integral is less than

$$
\left(\frac{3}{2}\right)^{(n-1) / 2} \int_{0}^{\alpha} t^{(n-1) / 2} e^{-t} d t \leq\left(\frac{3}{2}\right)^{(n-1) / 2} \int_{0}^{\infty} t^{(n-1) / 2} e^{-t} d t=\left(\frac{3}{2}\right)^{(n-1) / 2} \Gamma\left(\frac{n+1}{2}\right) ;
$$

while the second integral is less than

$$
\int_{\alpha}^{\infty} t^{(n-1) / 2}\left(\frac{t}{\alpha}+\frac{t}{2 \alpha}\right)^{(n-1) / 2} e^{-t} d t \leq\left(\frac{3}{2 \alpha}\right)^{(n-1) / 2} \int_{0}^{\infty} t^{n-1} e^{-t} d t=(n-1) !\left(\frac{3}{2 \alpha}\right)^{(n-1) / 2} .
$$

Adding these two estimates, we obtain the required inequality.

Proof of Theorem 1.2. The uniqueness of bounded solutions follows from Lemma 2.1. Let us show that $P_{\lambda}[f]$ is the bounded solution to (1.3). We begin with the proof of (1.4). By Fubini's theorem, translation and a change of variable we have

$$
\begin{aligned}
P_{\lambda}[1](x) & =x_{n} \int_{0}^{\infty}(4 \pi t)^{-n / 2} \exp \left(-\lambda^{2} t-\frac{x_{n}^{2}}{4 t}\right) \frac{d t}{t} \int_{\mathbb{R}^{n-1}} \exp \left(\frac{-\left|x^{\prime}-y^{\prime}\right|^{2}}{4 t}\right) d y^{\prime} \\
& =\frac{x_{n}}{2 \sqrt{\pi}} \int_{0}^{\infty} t^{-1 / 2} \exp \left(-\lambda^{2} t-\frac{x_{n}^{2}}{4 t}\right) \frac{d t}{t} \\
& =\frac{\lambda x_{n}}{2 \sqrt{\pi}} \int_{0}^{\infty} t^{-1 / 2} \exp \left(-t-\frac{\lambda^{2} x_{n}^{2}}{4 t}\right) \frac{d t}{t} \\
& =\frac{\lambda x_{n}}{2 \sqrt{\pi}} \cdot 2\left(\frac{\lambda x_{n}}{2}\right)^{-1 / 2} K_{1 / 2}\left(\lambda x_{n}\right)=\exp \left(-\lambda x_{n}\right),
\end{aligned}
$$

since $K_{1 / 2}(z)=(\pi / 2 z)^{1 / 2} \exp (-z)$ by $(2.1)$ with $v=1 / 2$.

Let $f$ be a bounded continuous function on $\mathbb{R}^{n-1}$. We have $\left|P_{\lambda}[f](x)\right| \leq\|f\|_{\infty} \exp \left(-\lambda x_{n}\right) \leq$ $\|f\|_{\infty}$ by (1.4). Since $P_{\lambda}(x)$ is a constant multiple of a derivative of the Green function $G_{\lambda}(x)$ for $-\Delta+\lambda^{2}$, it follows that $P_{\lambda}[f]$ satisfies the Helmholtz equation in $\mathbb{R}_{+}^{n}$. Finally, we claim

$$
\lim _{x \rightarrow x_{0}} P_{\lambda}[f](x)=f\left(x_{0}\right) \quad \text { for every } x_{0} \in \partial \mathbb{R}_{+}^{n} .
$$

Without loss of generality we may assume that $x_{0}=0$. For every $\varepsilon>0$ we find $\delta>0$ such that $\left|f\left(y^{\prime}\right)-f(0)\right|<\varepsilon$ for $\left|y^{\prime}\right|<2 \delta$ by the continuity of $f$. By (1.4) we have

$$
\begin{aligned}
\left|P_{\lambda}[f](x)-\exp \left(-\lambda x_{n}\right) f(0)\right| & \leq \int_{\mathbb{R}^{n-1}} P_{\lambda}\left(x ; y^{\prime}\right)\left|f\left(y^{\prime}\right)-f(0)\right| d y^{\prime} \\
& =\int_{\left|y^{\prime}\right|<2 \delta}+\int_{\left|y^{\prime}\right| \geq 2 \delta} P_{\lambda}\left(x ; y^{\prime}\right)\left|f\left(y^{\prime}\right)-f(0)\right| d y^{\prime} .
\end{aligned}
$$

The first integral is less than

$$
\int_{\left|y^{\prime}\right|<2 \delta} \varepsilon P_{\lambda}\left(x ; y^{\prime}\right) d y^{\prime} \leq \varepsilon \int_{\mathbb{R}^{n-1}} P_{\lambda}\left(x ; y^{\prime}\right) d y^{\prime}=\varepsilon \exp \left(-\lambda x_{n}\right) \leq \varepsilon ;
$$

while, by Lemma 2.2, the second integral is less than

$$
2\|f\|_{\infty} \int_{\left|y^{\prime}\right| \geq 2 \delta} P_{\lambda}\left(x ; y^{\prime}\right) d y^{\prime} \leq 2\|f\|_{\infty} C(\delta, \lambda, n) x_{n} \quad \text { for }|x|<\delta .
$$


Hence

$$
\limsup _{x \rightarrow 0}\left|P_{\lambda}[f](x)-f(0)\right|=\limsup _{x \rightarrow 0}\left|P_{\lambda}[f](x)-\exp \left(-\lambda x_{n}\right) f(0)\right| \leq \varepsilon .
$$

Since $\varepsilon>0$ is arbitrary, the claim follows. Thus $u=P_{\lambda}[f]$ satisfies (1.3). The proof is complete.

In the sequel, we freely use the elementary inequality

$$
t^{\alpha} \exp (-\beta t) \leq C(\alpha, \beta) \text { for } t>0,
$$

provided $\alpha, \beta>0$.

Proof of Theorem 1.3. In this proof all constants $C$ depend only on the dimension $n$. By translation we may assume that $x=\left(0, x_{n}\right)$. Let $y^{\prime} \in \mathbb{R}^{n-1}$ and $y=\left(y^{\prime}, 0\right) \in \partial \mathbb{R}_{+}^{n}$. Observe that $\lambda|x-y| \geq \lambda x_{n} \geq 1$ by assumption. If $\left|y^{\prime}\right| \geq \frac{4}{3} x_{n}$, then $|x-y| \geq \frac{1}{2}\left|y^{\prime}\right|+x_{n} \geq \max \left\{\frac{1}{2}\left|y^{\prime}\right|, x_{n}\right\}$ by an elementary calculation, so that

$$
\exp \left(\lambda x_{n}\right) P_{\lambda}\left(x ; y^{\prime}\right) \leq C \lambda^{(n-1) / 2} \frac{x_{n}}{\left(\left|y^{\prime}\right| / 2\right)^{(n+1) / 2}} \exp \left(-\frac{\lambda\left|y^{\prime}\right|}{2}\right)
$$

by Lemma 2.2. Hence

$$
\begin{aligned}
\exp \left(\lambda x_{n}\right) \int_{\left|y^{\prime}\right| \geq 4 x_{n} / 3} P_{\lambda}\left(x ; y^{\prime}\right) d y^{\prime} & \leq C \lambda^{(n-1) / 2} x_{n} \int_{\left|y^{\prime}\right| \geq 4 x_{n} / 3}\left|y^{\prime}\right|^{-(n+1) / 2} \exp \left(-\frac{\lambda\left|y^{\prime}\right|}{2}\right) d y^{\prime} \\
& =C \lambda^{(n-1) / 2} x_{n} \int_{4 x_{n} / 3}^{\infty} r^{(n-5) / 2} \exp \left(-\frac{\lambda r}{4}\right) \exp \left(-\frac{\lambda r}{4}\right) d r \\
& \leq C \lambda^{(n-1) / 2-(n-3) / 2} x_{n} \exp \left(-\frac{\lambda x_{n}}{3}\right) \int_{4 \lambda x_{n} / 3}^{\infty} r^{(n-5) / 2} \exp \left(-\frac{r}{4}\right) d r \\
& \leq C \lambda x_{n} \exp \left(-\frac{\lambda x_{n}}{3}\right) \int_{4 / 3}^{\infty} r^{(n-5) / 2} \exp \left(-\frac{r}{4}\right) d r .
\end{aligned}
$$

Since the last integral is convergent, it follows from (2.3) that

$$
\exp \left(\lambda x_{n}\right) \int_{\left|y^{\prime}\right| \geq 4 x_{n} / 3} P_{\lambda}\left(x ; y^{\prime}\right) d y^{\prime} \leq C \exp \left(-\frac{\lambda x_{n}}{4}\right) .
$$

On the other hand, if $a \leq\left|y^{\prime}\right| \leq \frac{4}{3} x_{n}$, then

$$
|x-y| \geq\left(x_{n}^{2}+a^{2}\right)^{1 / 2}=x_{n}\left(1+\left(\frac{a}{x_{n}}\right)^{2}\right)^{1 / 2} \geq x_{n}+\frac{x_{n}}{3}\left(\frac{a}{x_{n}}\right)^{2}=x_{n}+\frac{a^{2}}{3 x_{n}}
$$

by the elementary inequality $(1+t)^{1 / 2} \geq 1+\frac{1}{3} t$ for $0 \leq t \leq 3$, so that

$$
\exp \left(\lambda x_{n}\right) P_{\lambda}(x ; y) \leq C \lambda^{(n-1) / 2} x_{n}^{1-(n+1) / 2} \exp \left(-\frac{\lambda a^{2}}{3 x_{n}}\right)
$$

by Lemma 2.2. Hence

$$
\exp \left(\lambda x_{n}\right) \int_{a \leq\left|y^{\prime}\right| \leq 4 x_{n} / 3} P_{\lambda}\left(x ; y^{\prime}\right) d y^{\prime} \leq C\left(\lambda x_{n}\right)^{(n-1) / 2} \exp \left(-\frac{\lambda a^{2}}{3 x_{n}}\right) .
$$

This, together with (2.4), yields the required inequality. 


\section{The Helmholtz EQUation and harmonic MeAsure}

It is sufficient to construct a domain in $\mathbb{R}^{3}$ which fails the global boundary Harnack principle. Such a domain will be given by

$$
\{(x, y, z):(x, y) \in(-1,1) \times(-1,1), f(x, y)<z<1\}
$$

with a $\psi_{\alpha}$-Hölder continuous function $f$ on $(-1,1) \times(-1,1)$. The construction of $f$ is based on precise estimates of harmonic measures in the products $(-\varepsilon, \varepsilon) \times D$ with plane domains $D$ in $y z$-space. The correspondence $h(x, y, z)=\cos (\pi x /(2 \varepsilon)) u(y, z)$ leads to the Helmholtz equation

$$
\left(-\frac{\partial^{2}}{\partial y^{2}}-\frac{\partial^{2}}{\partial z^{2}}+\lambda^{2}\right) u(y, z)=0
$$

with $\lambda=\pi /(2 \varepsilon)$. For future references let us restate Theorems 1.2 and 1.3 as well as some results in Section 2 in this context.

Theorem 3.1. We have the following assertions:

(i) Let $D$ be an arbitrary domain in the yz-plane, $\mathbb{R}^{2}$. Suppose $u$ and $v$ are bounded solutions to (3.1) in D. If $u \leq v$ on $\partial D$, then $u \leq v$ in $D$.

(ii) Let $f$ be a bounded continuous function on $\mathbb{R}$. Then the boundary value problem (3.1) in the upper half plane $\mathbb{R}_{+}^{2}=\{(y, z): z>0\}$ and $u(y, 0)=f(y)$ for $y \in \mathbb{R}$ has a unique bounded solution

$$
P_{\lambda}[f](y, z)=\int_{-\infty}^{\infty} P_{\lambda}(y, z ; \eta) f(\eta) d \eta
$$

with

$$
P_{\lambda}(y, z ; \eta)=\frac{z}{4 \pi} \int_{0}^{\infty} \exp \left(-\lambda^{2} t-\frac{(y-\eta)^{2}+z^{2}}{4 t}\right) \frac{d t}{t^{2}} .
$$

(iii) $P_{\lambda}[1](y, z)=\exp (-\lambda z)$ for $(y, z) \in \mathbb{R}_{+}^{2}$; moreover,

$$
\int_{y}^{\infty} P_{\lambda}(y, z ; \eta) d \eta=\frac{1}{2} \exp (-\lambda z) \quad \text { for any } y \in \mathbb{R}
$$

(iv) Let $a>0$. If $(y, z) \in \mathbb{R}_{+}^{2}$ and $\lambda z \geq 1$, then

$$
\int_{y+a}^{\infty} P_{\lambda}(y, z ; \eta) d \eta \leq C \exp (-\lambda z) \cdot\left\{\exp \left(-\frac{\lambda z}{4}\right)+(\lambda z)^{1 / 2} \cdot \exp \left(-\frac{\lambda a^{2}}{3 z}\right)\right\}
$$

where $C$ is an absolute positive constant. In particular, if $\lambda \geq 1$, then

$$
\int_{y+a}^{\infty} P_{\lambda}(y, 1 ; \eta) d \eta \leq C \exp (-\lambda) \cdot\left\{\exp \left(-\frac{\lambda}{4}\right)+\lambda^{1 / 2} \cdot \exp \left(-\frac{\lambda a^{2}}{3}\right)\right\} .
$$

Remark 3.1. The second assertion of (iii) follows from symmetry. In (iv) the integral $\int_{y+a}^{\infty}$ can be replaced by $\int_{-\infty}^{y-a}+\int_{y+a}^{\infty}$. (Actually, this is more straightforward from Theorem 1.3.) If $a=0$, then the right hand side of the first inequality in (iv) is

$$
C \exp (-\lambda z) \cdot\left\{\exp \left(-\frac{\lambda z}{4}\right)+(\lambda z)^{1 / 2}\right\}
$$

This does not contradict (iii) since $\inf _{t>0}\left(\exp (-t / 4)+t^{1 / 2}\right)>0$. 
To facilitate the succeeding arguments we write $\omega_{\lambda}(E ; D)$ for the bounded solution $u$ to (3.1) in $D$ with $u=\chi_{E}$ on $\partial D$. This is the harmonic measure with respect to $-\Delta_{y z}+\lambda^{2}$, where $\Delta_{y z}$ is the Laplacian in the $y z$-plane. The value of $\omega_{\lambda}(E ; D)$ at $(y, z)$ is denoted by $\omega_{\lambda}^{(y, z)}(E ; D)$. For instance Theorem 3.1 (iii) and (iv) read

$$
\begin{aligned}
& \omega_{\lambda}^{(y, z)}\left(\partial \mathbb{R}_{+}^{2} ; \mathbb{R}_{+}^{2}\right)=\exp (-\lambda z), \quad \omega_{\lambda}^{(y, z)}\left((y, \infty) \times\{0\} ; \mathbb{R}_{+}^{2}\right)=\frac{1}{2} \exp (-\lambda z) ; \\
& \omega_{\lambda}^{(y, 1)}\left((y+a, \infty) \times\{0\} ; \mathbb{R}_{+}^{2}\right) \leq C \exp (-\lambda) \cdot\left\{\exp \left(-\frac{\lambda}{4}\right)+\lambda^{1 / 2} \cdot \exp \left(-\frac{\lambda a^{2}}{3}\right)\right\} .
\end{aligned}
$$

Let $\omega$ be the usual harmonic measure in $x y z$-space. Since $\cos (\lambda x) \omega_{\lambda}^{(y, z)}(E ; D)$ is harmonic in $(-\varepsilon, \varepsilon) \times D$ and vanishes on $\{-\varepsilon, \varepsilon\} \times D$ with $\varepsilon=\pi /(2 \lambda)$, it is easy to see that

$$
\cos (\lambda x) \omega_{\lambda}^{(y, z)}(E ; D) \leq \omega^{(x, y, z)}((-\varepsilon, \varepsilon) \times E ;(-\varepsilon, \varepsilon) \times D) \quad \text { in }(-\varepsilon, \varepsilon) \times D .
$$

In some cases, an opposite inequality (up to a multiplicative constant) holds for $(y, z)$ apart from $E$. To show such an inequality we start with a lower estimate of harmonic measure with respect to $-\Delta+\lambda^{2}$. We use the following notation. For $\varepsilon>0$ and $Y \in \mathbb{R}$ we let $S(Y, \varepsilon)=$ $(Y-\varepsilon, Y+\varepsilon) \times(0,2 \varepsilon)$ be the open square of center at $(Y, \varepsilon)$ with sides of length $2 \varepsilon$ and parallel to the coordinate axes. Let $S_{0}(Y, \varepsilon)=(Y-\varepsilon, Y+\varepsilon) \times\{0\}$ be the bottom side of $S(Y, \varepsilon)$.

Lemma 3.1. Let $\varepsilon>0$ and $\lambda=\pi /(2 \varepsilon)$. Then

$$
\omega_{\lambda}^{(Y, \varepsilon)}\left(S_{0}(Y, \varepsilon) ; S(Y, \varepsilon)\right) \geq \frac{\sinh (\sqrt{2} \pi / 2)}{\sinh (\sqrt{2} \pi)} .
$$

Proof. By translation we may assume that $Y=0$. It is easy to see that

$$
v(y, z)=\cos (\lambda y) \frac{\sinh (\sqrt{2} \lambda(2 \varepsilon-z))}{\sinh (2 \sqrt{2} \lambda \varepsilon)}
$$

satisfies $\left(-\Delta_{y z}+\lambda^{2}\right) v=0$ in $S(0, \varepsilon)$ and

$$
v(y, z)= \begin{cases}\cos (\lambda y) \leq 1 & \text { on } S_{0}(0, \varepsilon), \\ 0 & \text { on } \partial S(0, \varepsilon) \backslash S_{0}(0, \varepsilon) .\end{cases}
$$

Hence $\omega_{\lambda}\left(S_{0}(0, \varepsilon) ; S(0, \varepsilon)\right) \geq v$ in $S_{0}(0, \varepsilon)$. Evaluating at $(0, \varepsilon)$, we obtain

$$
\omega_{\lambda}^{(0, \varepsilon)}\left(S_{0}(0, \varepsilon) ; S(0, \varepsilon)\right) \geq \frac{\sinh (\sqrt{2} \lambda \varepsilon)}{\sinh (2 \sqrt{2} \lambda \varepsilon)}=\frac{\sinh (\sqrt{2} \pi / 2)}{\sinh (\sqrt{2} \pi)},
$$

as required.

Let us record the following scale invariant boundary Harnack principle on the open cube $Q(Y, \varepsilon)=(-\varepsilon, \varepsilon) \times S(Y, \varepsilon)$ of center at $(0, Y, \varepsilon)$ with sides of length $2 \varepsilon$ parallel to the coordinate axes. Although it is known that the scale invariant boundary Harnack principle holds for a Lipschitz domain, we can directly prove the following lemma since the domain is explicit.

Lemma 3.2. Let $\varepsilon>0$ and $Y \in \mathbb{R}$. If $u$ and $v$ are bounded positive harmonic functions in $Q(Y, \varepsilon)$ such that $u=v=0$ on $\{-\varepsilon, \varepsilon\} \times S(Y, \varepsilon)$, then

$$
\frac{u}{u(0, Y, \varepsilon)} \approx \frac{v}{v(0, Y, \varepsilon)} \quad \text { on }(-\varepsilon, \varepsilon) \times\{Y\} \times\{\varepsilon\},
$$

where the constant of comparison is independent of $u, v, Y$, and $\varepsilon$.

We have an inequality opposite to (3.4) up to a multiplicative constant. 
Lemma 3.3. Let $\varepsilon>0, \lambda=\pi /(2 \varepsilon)$ and $T=(-\varepsilon, \varepsilon) \times(-\infty, \infty)$. Then

$$
\omega^{(x, y, z)}(T \times\{0\} ; T \times(0, \infty)) \leq C_{0} \cos (\lambda x) \omega_{\lambda}^{(y, z)}\left(\partial \mathbb{R}_{+}^{2} ; \mathbb{R}_{+}^{2}\right)
$$

for $(x, y, z) \in T \times[\varepsilon, \infty)$, where $C_{0}$ is an absolute positive constant.

Proof. In view of the maximum principle, it suffices to show (3.5) on $T \times\{\varepsilon\}$. By the maximum principle

$$
\omega_{\lambda}\left(S_{0}(Y, \varepsilon) ; S(Y, \varepsilon)\right) \leq \omega_{\lambda}\left(\partial \mathbb{R}_{+}^{2} ; \mathbb{R}_{+}^{2}\right) \quad \text { in } S(Y, \varepsilon)
$$

for every $Y \in \mathbb{R}$. Let us apply Lemma 3.2 to $u=\omega(T \times\{0\} ; T \times(0, \infty))$ and $v(x, y, z)=$ $\cos (\lambda x) \omega_{\lambda}^{(y, z)}\left(S_{0}(Y, \varepsilon) ; S(Y, \varepsilon)\right)$. By Lemma 3.1 we have $u(0, Y, \varepsilon) \approx v(0, Y, \varepsilon)$, so that $u \approx v$ on $(-\varepsilon, \varepsilon) \times\{Y\} \times\{\varepsilon\}$. Hence, we have (3.5) on $(-\varepsilon, \varepsilon) \times\{Y\} \times\{\varepsilon\}$, and hence on $T \times\{\varepsilon\}$, since $Y \in \mathbb{R}$ is arbitrary.

We have a similar estimate for the harmonic measure of the semi-strip $T_{+}=(-\varepsilon, \varepsilon) \times(0, \infty)$.

Lemma 3.4. Let $\varepsilon>0, \lambda=\pi /(2 \varepsilon), T=(-\varepsilon, \varepsilon) \times(-\infty, \infty)$ and $T_{+}=(-\varepsilon, \varepsilon) \times(0, \infty)$. Then

$$
\omega^{(x, y, z)}\left(T_{+} \times\{0\} ; T \times(0, \infty)\right) \leq C \cos (\lambda x) \omega_{\lambda}^{(y, z)}\left((0, \infty) \times\{0\} ; \mathbb{R}_{+}^{2}\right)
$$

for $(x, y, z) \in T \times[\varepsilon, \infty)$, where $C$ is an absolute positive constant.

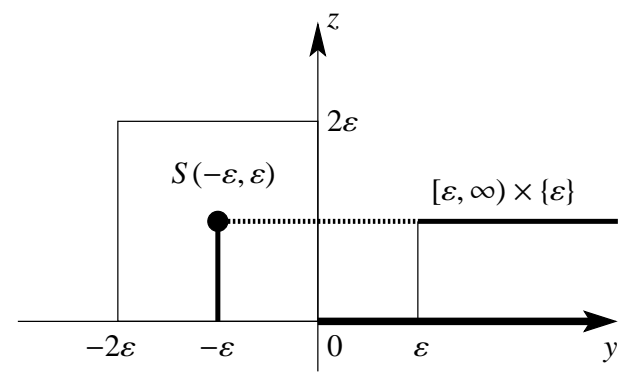

FIGURE 1. The projection of $T \times(0, \infty)$ onto the $y z$-plane.

Proof. See Figure 1 for the projection of $T \times(0, \infty)$ onto the $y z$-plane. Let $Y \in \mathbb{R}$. If $Y \geq \varepsilon$, then $(Y-\varepsilon, Y+\varepsilon) \subset(0, \infty)$, so that

$$
\omega_{\lambda}\left((0, \infty) \times\{0\} ; \mathbb{R}_{+}^{2}\right) \geq \omega_{\lambda}\left(S_{0}(Y, \varepsilon) ; S(Y, \varepsilon)\right) \quad \text { in } S(Y, \varepsilon)
$$

by the maximum principle. Hence

$$
\omega_{\lambda}\left((0, \infty) \times\{0\} ; \mathbb{R}_{+}^{2}\right) \geq \frac{\sinh (\sqrt{2} \pi / 2)}{\sinh (\sqrt{2} \pi)} \quad \text { on }[\varepsilon, \infty) \times\{\varepsilon\}
$$

by Lemma 3.1. Applying the interior Harnack inequality, we obtain

$$
\omega_{\lambda}\left((0, \infty) \times\{0\} ; \mathbb{R}_{+}^{2}\right) \geq C \text { on }[-\varepsilon, \infty) \times\{\varepsilon\},
$$

where $C$ is a positive absolute constant. In the same way as in the proof of Lemma 3.3 we obtain (3.6) on $(-\varepsilon, \varepsilon) \times[-\varepsilon, \infty) \times\{\varepsilon\}$ by using Lemma 3.2.

Let us show (3.6) on $(-\varepsilon, \varepsilon) \times\{-\varepsilon\} \times(0, \varepsilon)$. Let $Q(-\varepsilon, \varepsilon)=(-\varepsilon, \varepsilon) \times(-2 \varepsilon, 0) \times(0,2 \varepsilon)$ and $Q_{0}(-\varepsilon, \varepsilon)=(-\varepsilon, \varepsilon) \times(-2 \varepsilon, 0) \times\{0\}$. Observe that $u=\omega\left(T_{+} \times\{0\} ; T \times(0, \infty)\right)$ and $v(x, y, z)=$ $\cos (\lambda x) \omega_{\lambda}^{(y, z)}((0, \infty) \times\{0\} ;(-\infty, \infty) \times(0, \infty))$ are positive harmonic functions in $Q(-\varepsilon, \varepsilon)$ such that $u=v=0$ on $Q_{0}(-\varepsilon, \varepsilon) \cup\{-\varepsilon, \varepsilon\} \times(-2 \varepsilon, 0) \times(0,2 \varepsilon)$. Since $u(0,-\varepsilon, \varepsilon) \approx v(0,-\varepsilon, \varepsilon)$ by $(3.7)$, it follows from the scale invariant boundary Harnack principle that $u \approx v$ on $(-\varepsilon, \varepsilon) \times\{-\varepsilon\} \times(0, \varepsilon]$. 
Note that $u$ and $v$ vanish on the larger portion of the boundary than in Lemma 3.2 with $Y=-\varepsilon$, so that we have the comparison on the larger set $(-\varepsilon, \varepsilon) \times\{-\varepsilon\} \times(0, \varepsilon]$. Thus (3.6) holds on $(-\varepsilon, \varepsilon) \times\{-\varepsilon\} \times(0, \varepsilon]$. Since $u=v=0$ on $(-\varepsilon, \varepsilon) \times(-\infty,-\varepsilon) \times\{0\}$, it follows from the maximum principle that (3.6) holds in $(-\varepsilon, \varepsilon) \times(-\infty,-\varepsilon) \times(0, \infty) \cup(-\varepsilon, \varepsilon) \times[-\varepsilon, \infty) \times[\varepsilon, \infty)$, so in particular, in $T \times[\varepsilon, \infty)$.

Combining (3.3) and Lemma 3.4 with translation, we obtain the following corollary.

Corollary 3.1. Let $0<\varepsilon<1, \lambda=\pi /(2 \varepsilon), T=(-\varepsilon, \varepsilon) \times(-\infty, \infty)$ and $T(c)=(-\varepsilon, \varepsilon) \times(c, \infty)$ for $c \in \mathbb{R}$. If $|x|<\varepsilon$ and $y \leq c-a$ with $a \geq \varepsilon$, then

$$
\omega^{(x, y, 1)}(T(c) \times\{0\} ; T \times(-\infty, \infty)) \leq C_{1} \exp (-\lambda) \cdot\left\{\exp \left(-\frac{\lambda}{4}\right)+\lambda^{1 / 2} \cdot \exp \left(-\frac{\lambda a^{2}}{3}\right)\right\},
$$

where $C_{1}$ is an absolute positive constant.

We shall apply Corollary 3.1 with $\varepsilon \leq a \leq 1 / 2$ and with the roles of $y$ and $z$ interchanged. Note $\exp (-\lambda / 4) \leq \lambda^{1 / 2} \exp \left(-\lambda a^{2} / 3\right)$ in this case. For future reference we state it as a lemma.

Lemma 3.5. Let $0<\varepsilon<1, \lambda=\pi /(2 \varepsilon)$ and $\varepsilon \leq a \leq 1 / 2$. Let $R^{*}=(-\varepsilon, \varepsilon) \times(0, \infty) \times(-\infty, \infty)$ and $\widetilde{T}(c)=(-\varepsilon, \varepsilon) \times\{0\} \times(c, \infty)$. If $|x|<\varepsilon$ and $z \leq c-a$, then

$$
\omega^{(x, 1, z)}\left(\widetilde{T}(c) ; R^{*}\right) \leq 2 C_{1} \exp (-\lambda) \cdot \lambda^{1 / 2} \cdot \exp \left(-\frac{\lambda a^{2}}{3}\right) .
$$

\section{Proof of Theorem 1.1}

4.1. Construction of a wedge domain. Following the idea of [BB91] we construct a wedge domain which looks like a sharp ravine widening out rapidly. Let $n \geq 3$ be an integer and let $0<\varepsilon_{0}=\varepsilon_{1}<\varepsilon_{2}<\cdots<\varepsilon_{n}<1 / 2$. Let

$$
T_{n}=\operatorname{int}\left(\bigcup_{j=0}^{n}\left[-\varepsilon_{j}, \varepsilon_{j}\right] \times[j, j+1]\right), \quad W_{n}=T_{n} \times(-5,0) .
$$

With a slight abuse of notation, we denote the top of $W_{n}$ by $T_{n}$ as well. Let $S_{n}=\left(-\varepsilon_{n}, \varepsilon_{n}\right) \times\{n+$ $1\} \times(-5,0)$ be the right side of $W_{n}$. See Figure 2 . We shall show that the ratio of the harmonic measure of the top $T_{n}$ in $W_{n}$ evaluated at $p=(0,1,-4)$ and that of the side $S_{n}$ tends to 0 , i.e.,

$$
\frac{\omega^{p}\left(T_{n} ; W_{n}\right)}{\omega^{p}\left(S_{n} ; W_{n}\right)} \rightarrow 0 \quad \text { as } n \rightarrow \infty,
$$

provided $\left\{\varepsilon_{j}\right\}$ is suitably chosen. Actually, we shall give a stronger estimate. See (4.9) below.

With the aid of sharp estimates of the Poisson representation for the Helmholtz equation, we can take $\varepsilon_{j}$ explicitly. We specify $\varepsilon_{j}$ as

$$
\varepsilon_{n}=n^{-2} \quad \text { and } \quad \varepsilon_{n-i}=\varepsilon_{n}\left(12 n^{4}\right)^{-i} \quad \text { for } i=1, \ldots, n-1 .
$$

Let $\lambda_{j}=\pi /\left(2 \varepsilon_{j}\right)$ be the corresponding value for the Helmholtz equation. In view of (4.1) we have

and

$$
2 \lambda_{n}=\frac{\pi n^{2}}{2} \cdot 2<\frac{\pi n^{2}}{2} \cdot n^{2}=\frac{\pi n^{2}}{2} \cdot \frac{12 n^{4}}{12 n^{2}}=\frac{\lambda_{n-1}}{12 n^{2}} ;
$$

$$
\lambda_{n-i+1}+\cdots+\lambda_{n-1}+2 \lambda_{n}<\frac{\lambda_{n-i}}{12 n^{2}} \quad \text { for } i=2, \ldots, n-1
$$




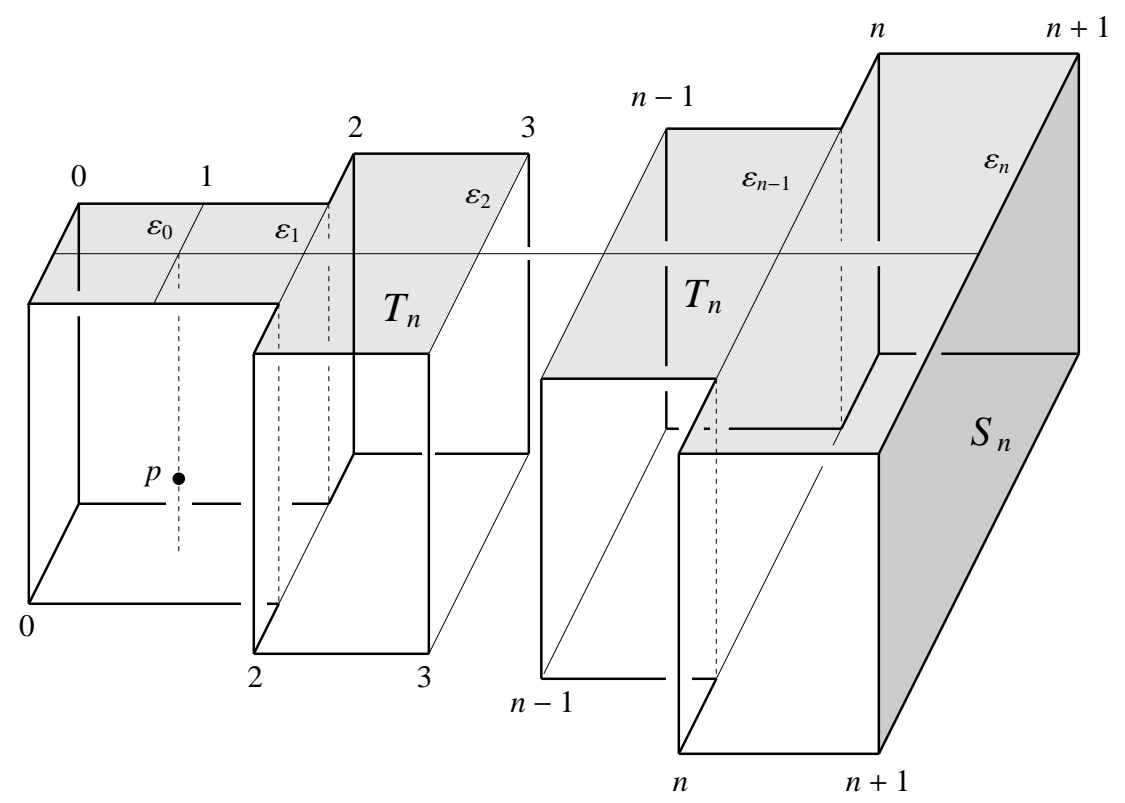

FIGURE 2. A wedge domain $W_{n}$ like a sharp ravine widening out rapidly.

In fact, if $2 \leq i \leq n-1$, then

$$
\begin{aligned}
\lambda_{n-i+1}+\cdots+\lambda_{n-1}+2 \lambda_{n} & =\frac{\pi n^{2}}{2} \cdot\left\{\left(12 n^{4}\right)^{i-1}+\cdots+\left(12 n^{4}\right)+2\right\} \\
& <\frac{\pi n^{2}}{2} \cdot\left(12 n^{4}\right)^{i-1} \cdot n<\frac{\pi n^{2}}{2} \cdot\left(12 n^{4}\right)^{i-1} \cdot n^{2}=\frac{\lambda_{n-i}}{12 n^{2}} .
\end{aligned}
$$

The explicit choice of $\varepsilon_{j}$ is crucial to give sharp modulus continuity in Theorem 1.1. This is a sharp contrast with [BB91].

Remark 4.1. Actually, $\varepsilon_{j}$ depends not only on $j$ but also on $n$, so it should be denoted by something like $\varepsilon_{j}^{n}$. However, the integer $n$ is fixed in the succeeding subsections, so the suffix $n$ is suppressed for simplicity.

4.2. Upper estimate of the harmonic measure of the top. We estimate $\Omega:=\omega\left(T_{n} ; W_{n}\right)$ inductively from the right. Let $W(j)=\left\{(x, y, z) \in W_{n}: y<j\right\}$ and $S(j)=\left\{(x, y, z) \in W_{n}: y=j\right\}$. (In this proof the integer $n$ is fixed, so the subscript " $n$ " is suppressed for simplicity.) Decompose $S(j)$ into $S^{\prime}(j) \cup S^{\prime \prime}(j)$ with

$$
S^{\prime}(j)=\left\{(x, y, z) \in S(j):-2-\frac{n-j}{n}<z<0\right\} \quad \text { and } \quad S^{\prime \prime}(j)=S(j) \backslash S^{\prime}(j) .
$$

See Figure 3.

We shall inductively estimate $M_{j}=\sup _{S^{\prime \prime}(j)} \Omega$ together with an auxiliary sequence $\left\{\widetilde{M}_{j}\right\}_{j}$ defined by $\widetilde{M}_{n}=M_{n} \exp \left(2 \lambda_{n}\right)$ and

$$
\widetilde{M}_{j}=M_{j} \exp \left(\lambda_{j}+\cdots+\lambda_{n-1}+2 \lambda_{n}\right) \text { for } j=1, \ldots, n-1 .
$$

Observe that $W_{n}=W(n+1), S_{n}=S(n+1)$, and

$$
W(j) \subset R(j) \subset R^{*}(j)
$$

with $R(j)=\left(-\varepsilon_{j-1}, \varepsilon_{j-1}\right) \times(0, j) \times(-5,0)$ and $R^{*}(j)=\left(-\varepsilon_{j-1}, \varepsilon_{j-1}\right) \times(-\infty, j) \times(-\infty, \infty)$ for $j=1, \ldots, n+1$. Let $E(j)=\left(-\varepsilon_{j-1}, \varepsilon_{j-1}\right) \times(0, j) \times\{0\}$ be the top of the rectangular cuboid $R(j)$. 
First, we estimate $M_{n}$. Let $\mathbb{R}_{-}^{2}=\{(y, z): z<0\}$. It follows from (4.3) with $j=n+1$ and the maximum principle that

$$
\Omega \leq \omega(E(n+1) ; R(n+1)) \leq \omega\left(\left(-\varepsilon_{n}, \varepsilon_{n}\right) \times \partial \mathbb{R}_{-}^{2} ;\left(-\varepsilon_{n}, \varepsilon_{n}\right) \times \mathbb{R}_{-}^{2}\right) \quad \text { in } W_{n} .
$$

Hence Lemma 3.3 and (3.2) upside down yield that

$$
\Omega(x, y, z) \leq C_{0} \exp \left(\lambda_{n} z\right) \quad \text { for }(x, y, z) \in W_{n} \text { with } z \leq-\varepsilon_{n} .
$$

In particular, $M_{n} \leq C_{0} \exp \left(-2 \lambda_{n}\right)$, in other words,

$$
\widetilde{M}_{n} \leq C_{0}
$$

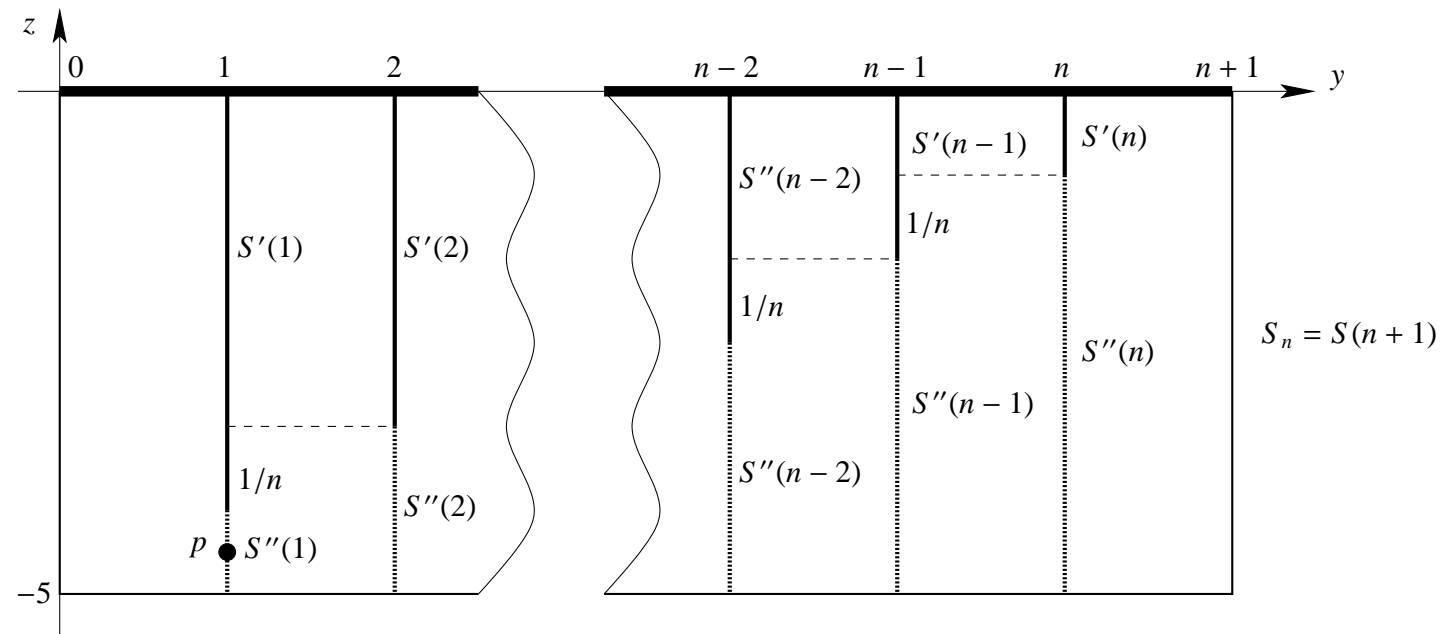

FIGURE 3. The projection of $W_{n}$ onto the $y z$-plane.

Secondly, we show an inductive inequality

$$
\widetilde{M}_{j-1} \leq\left(C_{0}+2 C_{1}\right)+C_{0} \widetilde{M}_{j} \text { for } j=2, \ldots, n .
$$

Let $2 \leq j \leq n$. It follows from (4.3) and the maximum principle that

$$
\Omega \leq \omega(E(j) ; R(j))+\omega\left(S^{\prime}(j) ; R(j)\right)+M_{j} \omega\left(S^{\prime \prime}(j) ; R(j)\right) \quad \text { in } W(j) .
$$

Let us estimate each term in the right hand side. By the same reasoning as above we have

$$
\omega(E(j) ; R(j)) \leq C_{0} \exp \left(-2 \lambda_{j-1}\right) \quad \text { on }\{(x, y, z) \in W(j): z \leq-2\} .
$$

By translation and Lemma 3.5 with $a=1 / n$ we have

$$
\omega^{(x, y, z)}\left(S^{\prime}(j) ; R(j)\right) \leq \omega^{(x, y, z)}\left(S^{*}(j) ; R^{*}(j)\right) \leq 2 C_{1} \exp \left(-\lambda_{j-1}\right) \cdot \lambda_{j-1}^{1 / 2} \cdot \exp \left(-\frac{\lambda_{j-1}}{3 n^{2}}\right)
$$

for $(x, y, z) \in S^{\prime \prime}(j-1)$, where $S^{*}(j)=\left(-\varepsilon_{j-1}, \varepsilon_{j-1}\right) \times\{j\} \times(-2-(n-j) / n, \infty)$. Combining (3.2) and Lemma 3.3 with $y$ and $z$ interchanged, we obtain $\omega\left(S^{\prime \prime}(j) ; R(j)\right) \leq C_{0} \exp \left(-\lambda_{j-1}\right)$ on $S(j-1)$. Hence, these three estimates altogether yield

$$
M_{j-1} \leq \exp \left(-\lambda_{j-1}\right)\left\{C_{0} \exp \left(-\lambda_{j-1}\right)+2 C_{1} \lambda_{j-1}^{1 / 2} \cdot \exp \left(-\frac{\lambda_{j-1}}{3 n^{2}}\right)+C_{0} M_{j}\right\} .
$$


Multiplying the both sides by $\exp \left(2 \lambda_{n}+\sum_{i=j-1}^{n-1} \lambda_{i}\right)$, we obtain

$$
\begin{aligned}
\widetilde{M}_{j-1} & \leq \exp \left(2 \lambda_{n}+\sum_{i=j}^{n-1} \lambda_{i}\right)\left\{C_{0} \exp \left(-\lambda_{j-1}\right)+2 C_{1} \lambda_{j-1}^{1 / 2} \cdot \exp \left(-\frac{\lambda_{j-1}}{3 n^{2}}\right)\right\}+C_{0} \widetilde{M}_{j} \\
& \leq\left(C_{0}+2 C_{1}\right)+C_{0} \widetilde{M}_{j},
\end{aligned}
$$

as required, since (4.2) with $i=n-j+1$ gives

$$
\begin{aligned}
& \exp \left(2 \lambda_{n}+\sum_{i=j}^{n-1} \lambda_{i}\right) \cdot \exp \left(-\lambda_{j-1}\right) \leq \exp \left(\frac{\lambda_{j-1}}{12 n^{2}}-\lambda_{j-1}\right) \leq 1 \\
& \exp \left(2 \lambda_{n}+\sum_{i=j}^{n-1} \lambda_{i}\right) \cdot \lambda_{j-1}^{1 / 2} \cdot \exp \left(-\frac{\lambda_{j-1}}{3 n^{2}}\right) \leq \lambda_{j-1}^{1 / 2} \cdot \exp \left(\frac{\lambda_{j-1}}{12 n^{2}}-\frac{\lambda_{j-1}}{3 n^{2}}\right) \leq 1 .
\end{aligned}
$$

Finally, rewriting (4.5) as

$$
\widetilde{M}_{j-1}+\frac{C_{0}+2 C_{1}}{C_{0}-1} \leq C_{0}\left(\widetilde{M}_{j}+\frac{C_{0}+2 C_{1}}{C_{0}-1}\right)
$$

and using (4.4), we obtain

$$
\widetilde{M}_{1}+\frac{C_{0}+2 C_{1}}{C_{0}-1} \leq C_{0}^{n-1}\left(\widetilde{M}_{n}+\frac{C_{0}+2 C_{1}}{C_{0}-1}\right) \leq C_{0}^{n-1}\left(C_{0}+\frac{C_{0}+2 C_{1}}{C_{0}-1}\right) \leq C_{2}^{n}
$$

with $C_{2}=C_{0}+\left(C_{0}+2 C_{1}\right) /\left(C_{0}-1\right)$. This means

$$
M_{1} \leq C_{2}^{n} \exp \left(-\lambda_{n}\right) \cdot \exp \left(-\sum_{j=1}^{n} \lambda_{j}\right)
$$

Since $p \in S^{\prime \prime}(1), \lambda_{j}=\pi /\left(2 \varepsilon_{j}\right)$ and $\varepsilon_{n}=n^{-2}$, it follows that

$$
\Omega(p) \leq C_{2}^{n} \exp \left(-\frac{\pi n^{2}}{2}\right) \cdot \exp \left(-\sum_{j=1}^{n} \frac{\pi}{2 \varepsilon_{j}}\right)=\exp \left(n \log C_{2}-\frac{\pi n^{2}}{2}\right) \cdot \exp \left(-\sum_{j=1}^{n} \frac{\pi}{2 \varepsilon_{j}}\right) .
$$

4.3. Lower estimate of the harmonic measure of the side. The lower estimate of the harmonic measure of the side is easier. It is based on an explicit positive harmonic function on a rectangular cuboid.

Lemma 4.1. Let $|\delta|<4 \varepsilon<1, R=(-\varepsilon, \varepsilon) \times(0,1+\delta) \times(-5,0)$ and let $\Sigma=(-\varepsilon, \varepsilon) \times\{1+\delta\} \times(-5,0)$ be the right side of $R$. Let

$$
h(x, y, z)=\frac{\sinh (\lambda y)}{\sinh (\lambda(1+\delta))} \cdot \cos \left(\frac{\pi x}{2 \varepsilon}\right) \cdot \sin \left(\frac{-\pi z}{5}\right) \text { with } \lambda=\frac{\pi}{2 \varepsilon} \sqrt{1+4(\varepsilon / 5)^{2}} .
$$

Then $h$ is the positive harmonic function in $R$ with boundary values

$$
h(x, y, z)= \begin{cases}\cos \left(\frac{\pi x}{2 \varepsilon}\right) \cdot \sin \left(\frac{-\pi z}{5}\right) & \text { on } \Sigma, \\ 0 & \text { on } \partial R \backslash \Sigma .\end{cases}
$$

Moreover,

$$
\omega^{(x, y, z)}(\Sigma ; R) \geq h(x, y, z) \geq C_{3} \frac{y}{\varepsilon} \cdot \exp \left(-\frac{\pi}{2 \varepsilon}\right) \cdot \cos \left(\frac{\pi x}{2 \varepsilon}\right) \cdot \sin \left(\frac{-\pi z}{5}\right) \quad \text { for }(x, y, z) \in R,
$$

where $C_{3}$ is an absolute positive constant. 
Proof. By an elementary calculation we see the first assertion. Hence the maximum principle yields the first inequality of the second assertion. Let us show the second inequality. By the definition of $\lambda$ and by the elementary inequality $1 \leq \sqrt{1+t} \leq 1+\frac{1}{2} t$ for $t \geq 0$ we have

$$
\frac{\pi}{2 \varepsilon} \leq \lambda \leq \frac{\pi}{2 \varepsilon}\left(1+2\left(\frac{\varepsilon}{5}\right)^{2}\right)
$$

so that

$$
\lambda(1+\delta) \leq \frac{\pi}{2 \varepsilon}\left\{1+|\delta|+2\left(\frac{\varepsilon}{5}\right)^{2}(1+|\delta|)\right\} \leq \frac{\pi}{2 \varepsilon}+3 \pi,
$$

since $|\delta|<4 \varepsilon<1$. By the elementary inequality $t \leq \sinh t \leq \frac{1}{2} \exp (t)$ for $t \geq 0$, we have

$$
\begin{aligned}
h(x, y, z) & \geq 2 \lambda y \cdot \exp \left(-\frac{\pi}{2 \varepsilon}-3 \pi\right) \cdot \cos \left(\frac{\pi x}{2 \varepsilon}\right) \cdot \sin \left(\frac{-\pi z}{5}\right) \\
& \geq \frac{\pi y}{\varepsilon} \cdot \exp (-3 \pi) \cdot \exp \left(-\frac{\pi}{2 \varepsilon}\right) \cdot \cos \left(\frac{\pi x}{2 \varepsilon}\right) \cdot \sin \left(\frac{-\pi z}{5}\right),
\end{aligned}
$$

which gives the required estimate with $C_{3}=\pi \exp (-3 \pi)$.

Now we construct a set smaller than $T_{n}$. Let

$$
\varepsilon_{j}^{\prime}=\varepsilon_{j}-\varepsilon_{j}^{2} \quad \text { and } \quad \lambda_{j}^{\prime}=\frac{\pi}{2 \varepsilon_{j}^{\prime}} \quad \text { for } j=0, \ldots, n,
$$

where $\varepsilon_{j}$ are as in (4.1). Define

$$
T_{n}^{\prime}=\operatorname{int}\left(\left[-\varepsilon_{n}^{\prime}, \varepsilon_{n}^{\prime}\right] \times\left[n+\varepsilon_{n-1}^{\prime}, n+1\right] \cup \bigcup_{j=0}^{n-1}\left[-\varepsilon_{j}^{\prime}, \varepsilon_{j}^{\prime}\right] \times\left[j+\varepsilon_{j-1}^{\prime}, j+1+\varepsilon_{j}^{\prime}\right]\right),
$$

$W_{n}^{\prime}=T_{n}^{\prime} \times(-5,0)$ and $S_{n}^{\prime}=\left(-\varepsilon_{n}^{\prime}, \varepsilon_{n}^{\prime}\right) \times\{n+1\} \times(-5,0)$. We give a lower estimate of $\omega\left(S_{n}^{\prime} ; W_{n}^{\prime}\right)$ inductively from the right. Let $\Sigma(j)=\left(-\varepsilon_{j-1}^{\prime}, \varepsilon_{j-1}^{\prime}\right) \times\left\{j+2 \varepsilon_{j-1}^{\prime}\right\} \times(-5,0)$ for $j=2, \ldots, n$. See Figure 4.

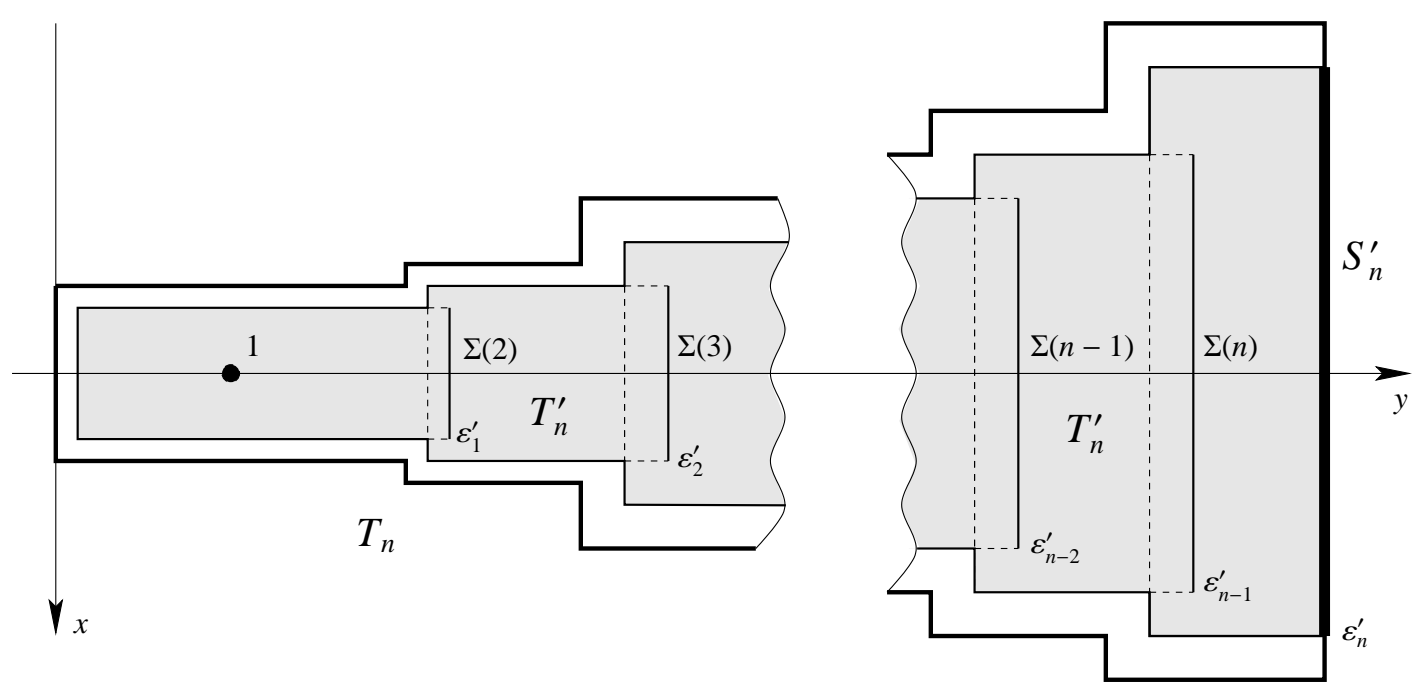

FIGURE 4. The projection of $W_{n}$ onto the $x y$-plane. 
Since $\left(-\varepsilon_{n}^{\prime}, \varepsilon_{n}^{\prime}\right) \times\left(n+\varepsilon_{n-1}^{\prime}, n+1\right) \times(-5,0) \subset W_{n}^{\prime}$, it follows from Lemma 4.1 with $\lambda=\lambda_{n}^{\prime}$, $\varepsilon=\varepsilon_{n}^{\prime}, \delta=-\varepsilon_{n-1}^{\prime}$, and the maximum principle that

$$
\begin{aligned}
\omega^{(x, y, z)}\left(S_{n}^{\prime} ; W_{n}^{\prime}\right) & \geq C_{3} \cdot \frac{\varepsilon_{n-1}^{\prime}}{\varepsilon_{n}^{\prime}} \cdot \exp \left(-\lambda_{n}^{\prime}\right) \cdot \cos \left(\lambda_{n}^{\prime} x\right) \cdot \sin \left(\frac{-\pi z}{5}\right) \\
& \geq C_{3} \cdot \frac{\varepsilon_{n-1}^{\prime}}{\varepsilon_{n}^{\prime}} \cdot \exp \left(-\lambda_{n}^{\prime}\right) \cdot \cos \left(\lambda_{n-1}^{\prime} x\right) \cdot \sin \left(\frac{-\pi z}{5}\right)
\end{aligned}
$$

for $(x, y, z) \in \Sigma(n)$. Since $\left(-\varepsilon_{n-1}^{\prime}, \varepsilon_{n-1}^{\prime}\right) \times\left(n-1+\varepsilon_{n-2}^{\prime}, n+2 \varepsilon_{n-1}^{\prime}\right) \times(-5,0) \subset W_{n}^{\prime}$, it follows from Lemma 4.1 with $\lambda=\lambda_{n-1}^{\prime}, \varepsilon=\varepsilon_{n-1}^{\prime}, \delta=2 \varepsilon_{n-1}^{\prime}-\varepsilon_{n-2}^{\prime}$, and the maximum principle that

$$
\omega^{(x, y, z)}\left(S_{n}^{\prime} ; W_{n}^{\prime}\right) \geq C_{3}^{2} \cdot \frac{\varepsilon_{n-2}^{\prime}}{\varepsilon_{n}^{\prime}} \cdot \exp \left(-\lambda_{n}^{\prime}\right) \cdot \exp \left(-\lambda_{n-1}^{\prime}\right) \cdot \cos \left(\lambda_{n-2}^{\prime} x\right) \cdot \sin \left(\frac{-\pi z}{5}\right)
$$

for $(x, y, z) \in \Sigma(n-1)$. Repeating this procedure, we obtain

$$
\omega^{(x, y, z)}\left(S_{n}^{\prime} ; W_{n}^{\prime}\right) \geq C_{3}^{n-1} \cdot \frac{\varepsilon_{1}^{\prime}}{\varepsilon_{n}^{\prime}} \cdot \cos \left(\lambda_{1}^{\prime} x\right) \cdot \sin \left(\frac{-\pi z}{5}\right) \cdot \exp \left(-\sum_{j=2}^{n} \lambda_{j}^{\prime}\right)
$$

for $(x, y, z) \in \Sigma(2)$. Finally, since $\left(-\varepsilon_{1}^{\prime}, \varepsilon_{1}^{\prime}\right) \times\left(1-\varepsilon_{1}^{\prime}, 2+2 \varepsilon_{1}^{\prime}\right) \times(-5,0) \subset W_{n}^{\prime}$. it follows from Lemma 4.1 with $\varepsilon=\varepsilon_{1}^{\prime}, \delta=3 \varepsilon_{1}^{\prime}$, and the maximum principle

$$
\omega^{(x, 1, z)}\left(S_{n}^{\prime} ; W_{n}^{\prime}\right) \geq C_{3}^{n} \cdot \frac{\varepsilon_{1}^{\prime}}{\varepsilon_{n}^{\prime}} \cdot \cos \left(\lambda_{1}^{\prime} x\right) \cdot \sin \left(\frac{-\pi z}{5}\right) \cdot \exp \left(-\sum_{j=1}^{n} \lambda_{j}^{\prime}\right) .
$$

Letting $x=0$ and $z=-4$, we obtain

$$
\omega^{p}\left(S_{n}^{\prime} ; W_{n}^{\prime}\right) \geq C_{3}^{n} \cdot \frac{\varepsilon_{1}^{\prime}}{\varepsilon_{n}^{\prime}} \cdot \sin \left(\frac{4 \pi}{5}\right) \cdot \exp \left(-\sum_{j=1}^{n} \lambda_{j}^{\prime}\right)
$$

where we recall $p=(0,1,-4)$. In view of (4.1) and (4.7) we see that $\varepsilon_{1}^{\prime}=\varepsilon_{1}-\varepsilon_{1}^{2}>\varepsilon_{1} / 2$, so that

$$
\frac{\varepsilon_{1}^{\prime}}{\varepsilon_{n}^{\prime}} \geq \frac{\varepsilon_{1}}{2 \varepsilon_{n}}=\frac{1}{2 \cdot\left(12 n^{4}\right)^{n-1}}
$$

and that

$$
\lambda_{j}^{\prime}=\frac{\pi}{2\left(\varepsilon_{j}-\varepsilon_{j}^{2}\right)}=\frac{\pi}{2}\left(\frac{1}{\varepsilon_{j}}+\frac{1}{1-\varepsilon_{j}}\right) \leq \frac{\pi}{2 \varepsilon_{j}}+\pi
$$

since $0<\varepsilon_{j}<1 / 2$. Hence, from (4.8) we have

$$
\omega^{p}\left(S_{n}^{\prime} ; W_{n}^{\prime}\right) \geq \sin \left(\frac{4 \pi}{5}\right) \cdot \frac{C_{3}^{n} \exp (-n \pi)}{2 \cdot\left(12 n^{4}\right)^{n-1}} \cdot \exp \left(-\sum_{j=1}^{n} \frac{\pi}{2 \varepsilon_{j}}\right) \geq \exp (-C n \log n) \cdot \exp \left(-\sum_{j=1}^{n} \frac{\pi}{2 \varepsilon_{j}}\right),
$$

where $C$ is an absolute positive constant. This, together with (4.6), yields

$$
\frac{\omega^{p}\left(T_{n} ; W_{n}\right)}{\omega^{p}\left(S_{n}^{\prime} ; W_{n}^{\prime}\right)} \rightarrow 0 \quad \text { as } n \rightarrow \infty
$$


4.4. Construction of a $\psi_{\alpha}$-Hölder domain. Let $0<\alpha<1$. Using $W_{n}$ and $W_{n}^{\prime}$ with translation and dilation, we construct a $\psi_{\alpha}$-Hölder domain which fails the global boundary Harnack principle. We write $r E$ for the dilation of a set $E$ by $r>0$. Observe from (4.1) that

$$
(n+1)^{-1} W_{n}^{\prime} \subset(n+1)^{-1} W_{n} \subset\left(-\frac{n^{-2}}{n+1}, \frac{n^{-2}}{n+1}\right) \times(0,1) \times\left(-\frac{5}{n+1}, 0\right) .
$$

Let $E_{n}=\left\{(x, y) \in \mathbb{R} \times(-\infty, 1):(x, y) \notin(n+1)^{-1} T_{n}\right\}$ and let $\ell_{n}$ be the distance between $E_{n}$ and $(n+1)^{-1} T_{n}^{\prime}$. Observe from (4.7) that

$$
\ell_{n}=(n+1)^{-1} \varepsilon_{1}^{2}=\frac{\left\{n^{-2}\left(12 n^{4}\right)^{1-n}\right\}^{2}}{n+1}=\frac{1}{12^{2(n-1)} n^{8 n-6}(n+1)} .
$$

Since $1 / \alpha>1$, if $n$ is sufficiently large, say $n \geq n_{0}$, then $\log \left(1 / \ell_{n}\right) \leq(n+1)^{1 / \alpha}$, so that

$$
(n+1)^{-1} \leq\left(\log \frac{1}{\ell_{n}}\right)^{-\alpha}=\psi_{\alpha}\left(\ell_{n}\right) .
$$

Define a function $F_{n}(x, y)$ on $\mathbb{R}^{2}$ by

$$
F_{n}(x, y):=\inf _{(\xi, \eta) \in E_{n}} \psi_{\alpha}(|(x, y)-(\xi, \eta)|) .
$$

Then $F_{n}(x, y) \geq(n+1)^{-1}$ on $(n+1)^{-1} T_{n}^{\prime}$. By definition $F_{n}(x, y) \geq 0$ on $\mathbb{R}^{2}$ and $F_{n}(x, y)=0$ on $E_{n}$. Moreover, $F_{n}(x, y)$ is $\psi_{\alpha}$-Hölder continuous. In fact, the concavity of $\psi_{\alpha}$ yields $\psi_{\alpha}(s+t) \leq$ $\psi_{\alpha}(s)+\psi_{\alpha}(t)$ for $s, t \geq 0$, and hence

$$
\psi_{\alpha}\left(\left|\left(x_{1}, y_{1}\right)-(\xi, \eta)\right|\right) \leq \psi_{\alpha}\left(\left|\left(x_{2}, y_{2}\right)-(\xi, \eta)\right|\right)+\psi_{\alpha}\left(\left|\left(x_{1}, y_{1}\right)-\left(x_{2}, y_{2}\right)\right|\right) .
$$

Taking the infimum for $(\xi, \eta) \in E_{n}$, we obtain

$$
F_{n}\left(x_{1}, y_{1}\right) \leq F_{n}\left(x_{2}, y_{2}\right)+\psi_{\alpha}\left(\left|\left(x_{1}, y_{1}\right)-\left(x_{2}, y_{2}\right)\right|\right),
$$

and changing the roles of $\left(x_{1}, y_{1}\right)$ and $\left(x_{2}, y_{2}\right)$,

$$
\left|F_{n}\left(x_{1}, y_{1}\right)-F_{n}\left(x_{2}, y_{2}\right)\right| \leq \psi_{\alpha}\left(\left|\left(x_{1}, y_{1}\right)-\left(x_{2}, y_{2}\right)\right|\right),
$$

as required.

Let $f_{n}(x, y)=\max \left\{-5(n+1)^{-1},-5 F_{n}(x, y)\right\}$ for $(x, y) \in \mathbb{R} \times(-\infty, 1)$. Then

(4.11) $\left|f_{n}\left(x_{1}, y_{1}\right)-f_{n}\left(x_{2}, y_{2}\right)\right| \leq 5 \psi_{\alpha}\left(\left|\left(x_{1}, y_{1}\right)-\left(x_{2}, y_{2}\right)\right|\right) \quad$ for $\left(x_{1}, y_{1}\right),\left(x_{2}, y_{2}\right) \in \mathbb{R} \times(-\infty, 1)$.

Moreover,

$$
\begin{aligned}
-5(n+1)^{-1} & \leq f_{n}(x, y) \leq 0 & & \text { in } \mathbb{R} \times(-\infty, 1), \\
f_{n}(x, y) & =-5(n+1)^{-1} & & \text { on }(n+1)^{-1} T_{n}^{\prime} \\
f_{n}(x, y) & =0 & & \text { on } E_{n} .
\end{aligned}
$$

Let $D_{n}=\left\{(x, y, z) \in \mathbb{R} \times(-\infty, 1) \times \mathbb{R}: f_{n}(x, y)<z<0\right\}$. Then

$$
\begin{aligned}
& (n+1)^{-1} W_{n}^{\prime} \subset D_{n} \subset(n+1)^{-1} W_{n}, \\
& \left\{(x, y, z) \in \partial D_{n}: z=0\right\} \subset(n+1)^{-1} T_{n}, \\
& \left\{(x, y, z) \in \partial D_{n}: y=1\right\} \supset(n+1)^{-1} S_{n}^{\prime} .
\end{aligned}
$$

Hence, (4.9), the maximum principle and scale invariance of harmonicity yield

$$
\frac{\omega^{p_{n}}\left(\left\{(x, y, z) \in \partial D_{n}: z=0\right\} ; D_{n}\right)}{\omega^{p_{n}}\left(\left\{(x, y, z) \in \partial D_{n}: y=1\right\} ; D_{n}\right)} \leq \frac{\omega^{p}\left(T_{n} ; W_{n}\right)}{\omega^{p}\left(S_{n}^{\prime} ; W_{n}^{\prime}\right)} \rightarrow 0 \quad \text { as } n \rightarrow \infty,
$$

where $p_{n}=\left(0,(n+1)^{-1},-4(n+1)^{-1}\right)$. 
Now let

$$
f(x, y)=\sum_{n=n_{0}}^{\infty} f_{n}\left(x-n^{-1}, y\right) \quad \text { for } y<1 .
$$

We see from (4.10) and (4.12) that

$$
\operatorname{supp}\left[f_{n}\left(x-n^{-1}, y\right)\right] \subset\left[\frac{1}{n}-\frac{n^{-2}}{n+1}, \frac{1}{n}+\frac{n^{-2}}{n+1}\right] \times[0,1],
$$

which implies that $\left\{\operatorname{supp}\left[f_{n}\left(x-n^{-1}, y\right)\right]\right\}_{n=n_{0}}^{\infty}$ are mutually disjoint. Hence (4.11) yields

$$
\left|f\left(x_{1}, y_{1}\right)-f\left(x_{2}, y_{2}\right)\right| \leq 5 \psi_{\alpha}\left(\left|\left(x_{1}, y_{1}\right)-\left(x_{2}, y_{2}\right)\right|\right) \quad \text { for }\left(x_{1}, y_{1}\right),\left(x_{2}, y_{2}\right) \in \mathbb{R} \times(-\infty, 1) .
$$

Finally, let $D=\{(x, y, z):|x|<1,|y|<1, f(x, y)<z<1\}$. This is a $\psi_{\alpha}$-Hölder domain, which is the union of a cuboid and countably many sharp ravines widening out rapidly. See Figure 5.

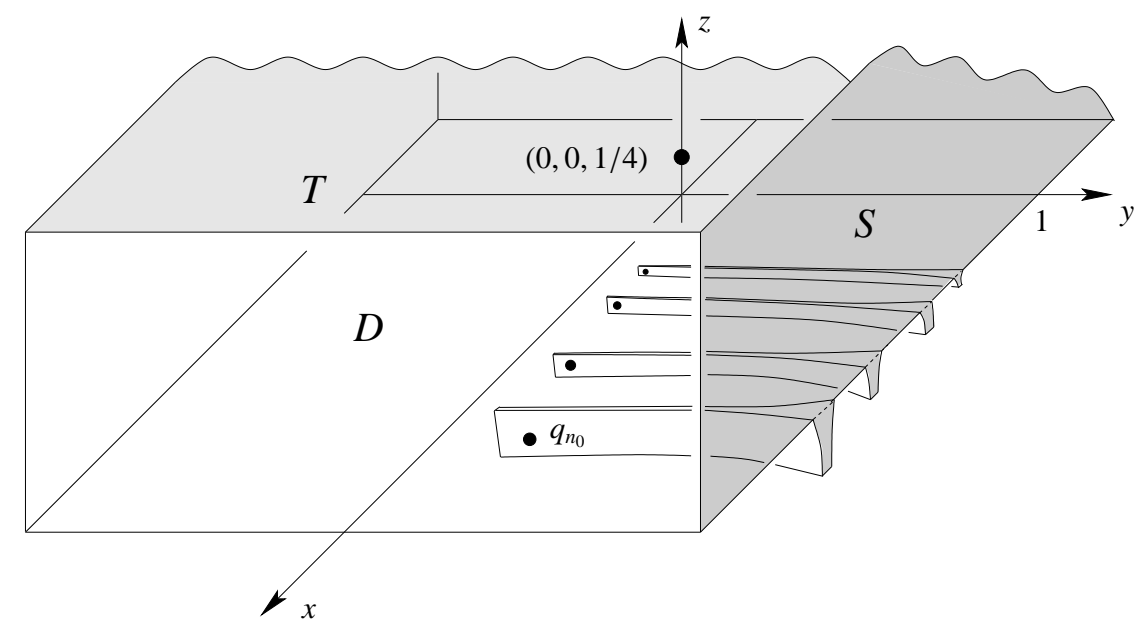

Figure 5. A domain which fails the global boundary Harnack principle.

Let $T=\{(x, y, z) \in \partial D: z=1\}$ be the top of $D$ and let $S=\{(x, y, z) \in \partial D: y=1\}$ be the right side of $D$. Then, it is easy to see that

$$
\omega^{(0,0,1 / 4)}(T ; D) \approx \omega^{(0,0,1 / 4)}(S ; D) \approx 1,
$$

while, with $q_{n}=\left(n^{-1},(n+1)^{-1},-4(n+1)^{-1}\right)$,

$$
\begin{aligned}
& \omega^{q_{n}}(T ; D) \leq \omega^{p_{n}}\left(\left\{(x, y, z) \in \partial D_{n}: z=0\right\} ; D_{n}\right), \\
& \omega^{q_{n}}(S ; D) \geq \omega^{p_{n}}\left(\left\{(x, y, z) \in \partial D_{n}: y=1\right\} ; D_{n}\right)
\end{aligned}
$$

by the maximum principle and translation. Hence (4.13) yields

$$
\frac{\omega^{q_{n}}(T ; D)}{\omega^{q_{n}}(S ; D)} \leq \frac{\omega^{p_{n}}\left(\left\{(x, y, z) \in \partial D_{n}: z=0\right\} ; D_{n}\right)}{\omega^{p_{n}}\left(\left\{(x, y, z) \in \partial D_{n}: y=1\right\} ; D_{n}\right)} \rightarrow 0 \quad \text { as } n \rightarrow \infty .
$$

This shows that $D$ fails the global boundary Harnack principle. In fact, let $V=(-1,1) \times$ $\left(-\frac{1}{2}, \frac{1}{2}\right) \times\left(-\frac{1}{2}, \frac{1}{2}\right)$ and $K=\left[-\frac{1}{2}, \frac{1}{2}\right] \times\left[-\frac{1}{3}, \frac{1}{3}\right] \times\left[-\frac{1}{3}, \frac{1}{3}\right]$. Then both $\omega(T ; D)$ and $\omega(S ; D)$ are positive harmonic functions in $D$, vanishing on $V \cap \partial D$ and yet for points $q_{n}$ and $(0,0,1 / 4)$ in $K \cap D$,

Thus Theorem 1.1 is proved.

$$
\frac{\omega^{q_{n}}(S ; D) / \omega^{(0,0,1 / 4)}(S ; D)}{\omega^{q_{n}}(T ; D) / \omega^{(0,0,1 / 4)}(T ; D)} \rightarrow \infty
$$




\section{APPENDIX}

For the reader's convenience we give a proof of (2.1) for $z=x>0$. We begin with a lemma.

Lemma 5.1. If $\alpha>0$, then

$$
\int_{0}^{\infty} \exp \left(-\alpha t-\frac{x^{2}}{4 t}\right) \frac{d t}{\sqrt{t}}=\sqrt{\frac{\pi}{\alpha}} \exp (-\sqrt{\alpha} x) \quad \text { for } x \geq 0 .
$$

Proof. By the change of variable $\alpha t=s$ we may assume that $\alpha=1$. Let $F(x)$ be the left hand side of (5.1) with $\alpha=1$. Then $F(0)=\Gamma(1 / 2)=\sqrt{\pi}$. If $x>0$, then the differentiation under the integral sign gives

$$
F^{\prime}(x)=-\int_{0}^{\infty} \frac{x}{2 t} \exp \left(-t-\frac{x^{2}}{4 t}\right) \frac{d t}{\sqrt{t}}
$$

Changing the variable $s=x^{2} /(4 t)$ and simplification yield

$$
F^{\prime}(x)=-\int_{\infty}^{0} \frac{x}{2 x^{2} /(4 s)} \exp \left(-\frac{x^{2}}{4 s}-s\right) \frac{1}{\sqrt{x^{2} /(4 s)}}\left(-\frac{x^{2}}{4 s^{2}}\right) d s=-F(x) .
$$

Solving this differential equation, we obtain (5.1) for $\alpha=1$

Proof of (2.1). Let $v>-1 / 2$ and $x>0$. First we note

$$
t^{-(v+1 / 2)}=\frac{1}{\Gamma(v+1 / 2)} \int_{0}^{\infty} e^{-t s} s^{v-1 / 2} d s \text { for } t>0,
$$

which can be easily derived from the change of variable $u=t s$ and the definition of the Gamma function. Plugging this identity in the definition of $K_{v}(x)$ and using Fubini's theorem, we obtain

$$
K_{v}(x)=\frac{1}{2}\left(\frac{x}{2}\right)^{v} \frac{1}{\Gamma(v+1 / 2)} \int_{0}^{\infty} s^{\nu-1 / 2} d s \int_{0}^{\infty} \exp \left(-(1+s) t-\frac{x^{2}}{4 t}\right) \frac{d t}{\sqrt{t}}
$$

By (5.1) with $\alpha=1+s$ and by the change of variable $t=(1+s)^{1 / 2}$ we have

$$
\begin{aligned}
K_{v}(x) & =\frac{1}{2}\left(\frac{x}{2}\right)^{v} \frac{1}{\Gamma(v+1 / 2)} \int_{0}^{\infty} s^{\nu-1 / 2}\left(\frac{\pi}{1+s}\right)^{1 / 2} \exp \left(-(1+s)^{1 / 2} x\right) d s \\
& =\left(\frac{x}{2}\right)^{v} \frac{\sqrt{\pi}}{\Gamma(v+1 / 2)} \int_{1}^{\infty}\left(t^{2}-1\right)^{\nu-1 / 2} \exp (-t x) d t .
\end{aligned}
$$

Again we make use the change of variable $s=(t-1) x$. Noting $t=1+s / x$ and $t^{2}-1=\frac{2 s}{x}\left(1+\frac{s}{2 x}\right)$, we obtain

$$
\begin{aligned}
K_{v}(x) & =\left(\frac{x}{2}\right)^{v} \frac{\sqrt{\pi}}{\Gamma(v+1 / 2)} \int_{0}^{\infty}\left\{\frac{2 s}{x}\left(1+\frac{s}{2 x}\right)\right\}^{v-1 / 2} \exp (-x-s) \frac{d s}{x} \\
& =\left(\frac{x}{2}\right)^{v} \frac{\sqrt{\pi}}{\Gamma(v+1 / 2)}\left(\frac{2}{x}\right)^{v-1 / 2} \frac{e^{-x}}{x} \int_{0}^{\infty} s^{v-1 / 2}\left(1+\frac{s}{2 x}\right)^{v-1 / 2} \exp (-s) d s,
\end{aligned}
$$

which leads to (2.1) after a simplification. 


\section{REFERENCES}

[Aik01] H. Aikawa, Boundary Harnack principle and Martin boundary for a uniform domain, J. Math. Soc. Japan 53 (2001), no. 1, 119-145. 1, 2

[Aik04] _ Potential-theoretic characterizations of nonsmooth domains, Bull. London Math. Soc. 36 (2004), no. 4, 469-482. 1, 2

[Aik14] _ Extended Harnack inequalities with exceptional sets and a boundary Harnack principle, J. Anal. Math. 124 (2014), 83-116. 2, 3

[Aik15] _ Intrinsic ultracontractivity via capacitary width, Rev. Mat. Iberoam. 31 (2015), no. 3, 10411106. 2

[Anc78] A. Ancona, Principe de Harnack à la frontière et théorème de Fatou pour un opérateur elliptique dans un domaine lipschitzien, Ann. Inst. Fourier (Grenoble) 28 (1978), no. 4, 169-213. 1, 2

[BB91] R. F. Bass and K. Burdzy, A boundary Harnack principle in twisted Hölder domains, Ann. of Math. (2) 134 (1991), no. 2, 253-276. 1, 2, 3, 11, 12

[BB92] _ Lifetimes of conditioned diffusions, Probab. Theory Related Fields 91 (1992), no. 3-4, 405443. 2

[BB93] 는 The Martin boundary in non-Lipschitz domains, Trans. Amer. Math. Soc. 337 (1993), no. 1, $361-378.3$

[BB94] _ The boundary Harnack principle for nondivergence form elliptic operators, J. London Math. Soc. (2) 50 (1994), no. 1, 157-169. 1, 3

[BBB91] R. Bañuelos, R. F. Bass, and K. Burdzy, Hölder domains and the boundary Harnack principle, Duke Math. J. 64 (1991), no. 1, 195-200. 1, 2

[Bog97] K. Bogdan, The boundary Harnack principle for the fractional Laplacian, Studia Math. 123 (1997), no. $1,43-80.1$

[CFMS81] L. A. Caffarelli, E. Fabes, S. Mortola, and S. Salsa, Boundary behavior of nonnegative solutions of elliptic operators in divergence form, Indiana Univ. Math. J. 30 (1981), no. 4, 621-640. 3

[Dah77] B. E. J. Dahlberg, Estimates of harmonic measure, Arch. Rational Mech. Anal. 65 (1977), no. 3, 275288. 1

[JK82] D. S. Jerison and C. E. Kenig, Boundary behavior of harmonic functions in nontangentially accessible domains, Adv. in Math. 46 (1982), no. 1, 80-147. 1, 2

[SW99] R. Song and J.-M. Wu, Boundary Harnack principle for symmetric stable processes, J. Funct. Anal. 168 (1999), no. 2, 403-427. 1

[Wat95] G. N. Watson, A treatise on the theory of Bessel functions, Cambridge Mathematical Library, Cambridge University Press, Cambridge, 1995, Reprint of the second (1944) edition. 5

[Wu78] J. M. G. Wu, Comparisons of kernel functions, boundary Harnack principle and relative Fatou theorem on Lipschitz domains, Ann. Inst. Fourier (Grenoble) 28 (1978), no. 4, 147-167. 1, 2

Department of Mathematics, Hokkaido University, Sapporo 060-0810, Japan

E-mail address: aik@math.sci.hokudai .ac.jp 